\title{
The Genetics of Parkinson Disease: To Test or Not to Test
}

Can. J. Neurol. Sci. 2007; 34: 266-267

Parkinson's Disease (PD) is one of the commonest neurodegenerative disorders, affecting 1 in 100 individuals over the age of 60 . Since its original description in the mid-19th century, great strides have been made in the understanding of the pathology and biochemical abnormalities. The introduction of levodopa for symptomatic treatment in the 1960's, resulted in significant improvement in motor function and quality of life.

One of the remaining mysteries is the etiology. Previously, the focus has been on environmental factors and toxins, such as viruses, manganese and pesticides. A variety of factors such as living in a rural area, mild head injury, and lack of smoking have been reported to increase the risk of PD. ${ }^{1}$ However, with increasing life expectancy, it has become evident that at least $10 \%$ of patients with PD have similarly affected relatives. Over the past 2 decades, research has shifted to the identification of genetic factors.

The first major breakthrough came in 1997 with the report of a large multi-generation family with Parkinsonism, and subsequent isolation of the responsible gene (now known as SNCA) coding for $\propto$-synuclein. ${ }^{2}$ This was a novel finding, and changed the nomenclature of the Parkinsonian conditions, leading to PD being identified as a synucleinopathy. (Synucleinopathies now encompass both sporadic and SCNAPD, Lewy body disease, and multi-system atrophy).

Over the past ten years, eight Mendelian genes have been identified to cause Parkinsonism. These have been labeled PARK 1-9 (PARK 4 is homologous to PARK 1). ${ }^{3}$ Parkin (PARK 2 ), inherited as an autosomal recessive trait, appears to be the most common cause of early onset of PD, occurring in up to $20 \%$ of cases. The protein is thought to be involved in protein degradation in the ubiquitin proteosomal system. PARK 6 (PINK1) and 7 (DJ1 gene), both autosomal recessive, are uncommon; the gene products may be involved in stabilizing mitochondrial function. Initial reports suggested that heterozygote carriers of these recessive mutations may be at higher risk for developing "typical" PD in later life. This remains controversial and recent reappraisal of the data suggests that this is not the case.

In 2004, the LRRK 2 (PARK 8) gene was discovered, and mutations at this locus shown to be a common cause of Parkinsonism both is familial and sporadic cases. ${ }^{4}$

The LRRK 2 mutations can cause what appears to be typical PD with late onset, and responsiveness to levolopa. However, even in the same family, manifestations can be variable as some individuals have very late onset and little progression; in others, the gene is non penetrant. Occasionally, dementia or atypical Parkinsonism may be seen. On autopsy, pathology can also be variable.
The LRRK 2 gene is a very large gene consisting of 51 exons, with pathological mutations reported to date in 10 of the exons. The most common mutation is G2019S, located in exon 41. The frequency of this mutation varies among different ethnic groups, with the highest frequency found in PD patients from Northern Africa, including the Arabic (30\%) and Ashkenazy Jewish (18\%) populations. It has been postulated that the founder mutation (the same one for both populations) occurred over 3,000 years ago. This mutation is much less common in the Northern European populations. Up to $1 \%$ of patients with early onset PD carry this gene.

Thus, based on population data, it appears that the two most common involved loci in familial and sporadic PD are the genes for Parkin and LRRK 2. Until very recently, genetic testing was only available in research laboratories under research protocols, but has now become available as a service in the U.S. Should testing be routinely available in Canada? Several general issues need to be considered:

1) If individuals are confirmed to have a mutated gene, treatment remains the same. There is no neuroprotective therapy or impact on management.

2) Penetrance is decreased in LRRK 2, that is, not everyone who has this gene will develop symptoms. Alternatively, some individuals may be quite elderly before showing symptoms. Thus, clinical correlation can be difficult to determine.

3) Only the more common mutations can be easily identified. Sequencing of the whole gene to identify less common mutations is very expensive and time consuming. 4) As these two genetic loci still represent a minority of PD cases, cost-effectiveness of testing would be low.

4) Heterozygote carriers of the Parkin mutation are not uncommon. However, significant controversy exists on whether this translates to a predisposition for developing PD (recent evidence suggests not).

5) Psychosocial effects of testing in the PD population are unknown.

The two papers in this issue of the Journal address the issue of whether LRRK2 is a cause of PD in the Canadian population with emphasis on French Canadians. A total of 335 patients with PD from Ontario and Quebec were screened; of these, the majority (252) were drawn from the French Canadian population. ${ }^{5,6}$ Both papers conclude that the common mutation (G2019S) is not present in this population. Dupre et $\mathrm{al}^{5}$ additionally screened exon 31 , while Grimes et $\mathrm{al}^{6}$ screened a total of seven exons with negative results. Concomitant genetic 
screening in 224 normal controls also did not reveal any mutations. Thus, it appears that in this population, even excluding the factors listed above, testing for LRRK 2 mutations would not be a fruitful diagnostic exercise.

Is there a place for any genetic testing? In individuals with atypical symptoms, for example cerebellar ataxia, or a family history, it is important to remember the autosomal dominant triplet repeat conditions that can mimic PD. These include the spinocerebellar ataxias types 2, 3, 6 and 17, and late onset Huntington disease. Genetic testing is readily available, and should be considered both for diagnosis, and family counseling.

In conclusion, the search for etiological factors for PD continues. In a minority of patients, there may be a significant genetic cause. In the majority, the etiology continues to likely be multi-factorial, due to a genetic predisposition and one or more as yet unidentified environmental factors. Further research hopefully will lead to a better understanding of pathogenesis, and to a preventative or neuroprotective therapy. In the meantime, genetic testing and screening for PD associated genes should remain in the research laboratory.

\section{REFERENCES}

1. Semchuk KM, Love EJ, Lee RG. Parkinson's disease: a test of the multifactorial etiologic hypothesis. Neurology. 1993;43(6): 1173-80.

2. Polymeropoulos MH, lavedan C, Leroyt E, Ide SE, Dehejia A, Dutra A, et al. Mutation in the $\alpha$-synuclein gene identified in families with Parkinson's disease. Science. 1997;276:2045-7.

3. Klein C, Schlossmacher MG. The genetics of Parkinson disease: implications for neurological care. Nat Clin Prac Neurol. March 2006; 2:136-46

4. Zimprich A, Bisup S, Leitner P, Lichtner P, Farrer M, Lincoln S, et al. Mutations in LRRK2 cause autosomal-dominant parkinsonism with pleomorphic pathology. Neuron. 2004; 44:601-7.

5. Dupré N, Rivière J, Myers RM, Provencher P, Pourcher E, Emond F, Rouleau GA. LRRK2 is not a significant cause of Parkinson's disease in French-Canadians. Can J Neurol Sci. 2007;34:333-5.

6. Grimes DA, Racacho L, Han F, Panisset M, Bulman DE. LLRK2 Screening in a Canadian Parkinson's disease cohort. Can J Neurol Sci. 2007;34:336-8.
Oksana Suchowersky Calgary, Alberta 